\title{
A PARABOLIC SINGULAR INTEGRAL OPERATOR WITH ROUGH KERNEL
}

\author{
YANPING CHEN, YONG DING ${ }^{\circledR}$ and DASHAN FAN
}

(Received 10 April 2006; accepted 15 September 2006)

Communicated by A. J. Pryde

Abstract

Let $\Omega\left(y^{\prime}\right)$ be an $H^{1}\left(S^{n-1}\right)$ function on the unit sphere satisfying a certain cancellation condition. We study the $L^{p}$ boundedness of the singular integral operator

$$
T f(x)=\text { p.v. } \int_{\mathbb{R}^{n}} f(x-y) \Omega\left(y^{\prime}\right) \rho(y)^{-\alpha} d y,
$$

where $\alpha \geq n$ and $\rho$ is a norm function which is homogeneous with respect to certain nonistropic dilation. The result in the paper substantially improves and extends some known results.

2000 Mathematics subject classification: 42B20, 42B25.

Keywords and phrases: parabolic singular integral, Hardy space, rough kernel.

\section{Introduction}

Let $\mathbb{R}^{n}$ be the $n$-dimensional Euclidean space with the routine norm $|x|$ for each $x \in \mathbb{R}^{n}$. Let $S^{n-1}=\left\{x \in \mathbb{R}^{n}:|x|=1\right\}$ be the unit sphere on $\mathbb{R}^{n}$ equipped with the Lebesgue measure $\sigma\left(x^{\prime}\right)$, where we use $x^{\prime}$ to denote the unit vector in the direction of $x$. To study the existence and regularity results for an elliptic differential operator, that is,

$$
D=\sum_{i, j=1}^{n} a_{i, j} \frac{\partial^{2}}{\partial x_{i} \partial x_{j}}
$$

with constant coefficients $\left\{a_{i, j}\right\}$, among some other estimates, one needs to study the singular integral operator $T$ with a convolution kernel $K$ (see [1] or [2]) satisfying:

(a) $K\left(\mu x_{1}, \ldots, \mu x_{n}\right)=\mu^{-n} K(x)$, for any $\mu>0$;

(b) $K \in C^{\infty}\left(\mathbb{R}^{n} \backslash\{0\}\right)$;

(c) $\int_{S^{n-1}} K\left(x^{\prime}\right) d \sigma\left(x^{\prime}\right)=0$.

The research was supported by NSF of China (Grant: 19371046 and 10571015) and SRFDP of China (Grant: 20050027025).

(c) 2008 Australian Mathematical Society 1446-7887/08 \$A2.00+0.00 
Similarly, for the heat operator

$$
D=\frac{\partial}{\partial x_{1}}-\sum_{j=2}^{n} \frac{\partial^{2}}{\partial x_{j}^{2}},
$$

the corresponding singular integral operator $T$ have a kernel $K$ satisfying:

(a') $K\left(\mu^{2} x_{1}, \ldots, \mu x_{n}\right)=\mu^{-n-1} K(x)$, for any $\mu>0$;

(b') $K \in C^{\infty}\left(\mathbb{R}^{n} \backslash\{0\}\right)$;

(c') $\int_{S^{n-1}} K\left(x^{\prime}\right)\left(2 x_{1}^{\prime 2}+x_{2}^{\prime 2}+\cdots+x_{n}^{\prime 2}\right) d \sigma\left(x^{\prime}\right)=0$.

To study a more general parabolic differential operator with constant coefficients, in 1966, Fabes and Rivière [6] defined the parabolic singular integral operator

$$
T f(x)=\text { p.v. } \int_{\mathbb{R}^{n}} K(y) f(x-y) d y,
$$

with $K$ satisfying:

(A) $K\left(\mu^{\alpha_{1}} x_{1}, \ldots, \mu^{\alpha_{n}} x_{n}\right)=\mu^{-\alpha} K\left(x_{1}, \ldots, x_{n}\right), \mu>0, \alpha=\sum_{i=1}^{n} \alpha_{i}$;

(B) $K \in C^{\infty}\left(\mathbb{R}^{n} \backslash\{0\}\right)$;

(C) $\int_{S^{n-1}} K\left(x^{\prime}\right) J\left(x^{\prime}\right) d \sigma\left(x^{\prime}\right)=0$;

where $\alpha_{i} \geq 1(i=1,2, \ldots, n)$ and $J\left(x^{\prime}\right)=\alpha_{1} x_{1}^{\prime 2}+\cdots+\alpha_{n} x_{n}^{\prime 2}$ is shown as follows. For any $x \in \mathbb{R}^{n}$, set

$$
\begin{aligned}
x_{1}= & \rho^{\alpha_{1}} \cos \varphi_{1} \cdots \cos \varphi_{n-2} \cos \varphi_{n-1} \\
x_{2}= & \rho^{\alpha_{2}} \cos \varphi_{1} \cdots \cos \varphi_{n-2} \sin \varphi_{n-1} \\
& \vdots \\
x_{n-1}= & \rho^{\alpha_{n-1}} \cos \varphi_{1} \sin \varphi_{2} \\
x_{n}= & \rho^{\alpha_{n}} \sin \varphi_{1} .
\end{aligned}
$$

Then $d x=\rho^{\alpha-1} J\left(x^{\prime}\right) d \rho d \sigma\left(x^{\prime}\right)$ and $\rho^{\alpha-1} J\left(x^{\prime}\right)$ is the Jacobian of the above transformation. One may check that $J\left(x^{\prime}\right)$ is a $C^{\infty}$ function on $S^{n-1}$, that is, bounded below uniformly by 1 . Moreover, without loss of generality, we may assume that $\alpha_{n} \geq \alpha_{n-1} \geq \cdots \geq \alpha_{1} \geq 1$. Note that the above condition (A) can be written as:

$\left(\mathrm{A}^{\prime}\right) \quad K\left(A_{\mu} x\right)=\left|\operatorname{det}\left(A_{\mu}\right)\right|^{-1} K(x)$;

where $A_{\mu}=\operatorname{diag}\left[\mu^{\alpha_{1}}, \ldots, \mu^{\alpha_{n}}\right]$ is a diagonal matrix.

For each fixed $x \in \mathbb{R}^{n}$, the function

$$
F(x, \rho)=\sum_{i=1}^{n} \frac{x_{i}^{2}}{\rho^{2 \alpha_{i}}},
$$

is a strictly decreasing function of $\rho>0$. Therefore, there exists an unique $\rho=\rho(x)$ for which $F(x, \rho)=1$. It was proved in [6] that $\rho$ is a metric on $\mathbb{R}^{n}$. Furthermore, we 
observe that $\left(\mathbb{R}^{n}, \rho\right)$ is a homogeneous group that admits a family of dilations $\delta_{\mu}$ $=\exp (A \log \mu)$ for which $\rho\left(\delta_{\mu} x\right)=\mu \rho(x), \mu>0$, where $A$ is a diagonalizable linear operator with positive eigenvalues.

By the above coordinates of polar type, one now has that

$$
T f(x)=\text { p.v. } \int_{\mathbb{R}^{n}} f(x-y) \Omega\left(y^{\prime}\right) \rho(y)^{-\alpha} d y,
$$

where $\Omega\left(y^{\prime}\right)=K\left(y^{\prime}\right)$ satisfying

$$
\int_{S^{n-1}} \Omega\left(y^{\prime}\right) J\left(y^{\prime}\right) d \sigma\left(y^{\prime}\right)=0 .
$$

The following theorem was proved by Fabes and Riviere in [6].

THEOREM A. If $\Omega \in C^{1}\left(S^{n-1}\right)$ and satisfies (1.1), then the operator $T$ is bounded on $L^{p}\left(\mathbb{R}^{n}\right)$ for $1<p<\infty$.

Later, the above theorem was improved by Nagel et al. [9] and the regularity condition on $\Omega$ was removed. The result is the following.

THEOREM B. If $\Omega \in L \log ^{+} L\left(S^{n-1}\right)$ and satisfies (1.1), then the operator $T$ is bounded on $L^{p}\left(\mathbb{R}^{n}\right)$ for $1<p<\infty$.

On the other hand, it was shown in [10] that in the case $\alpha_{1}=\alpha_{2}=\cdots=\alpha_{n}=1$, the condition $\Omega \in L \log ^{+} L\left(S^{n-1}\right)$ can be replaced further by the weaker condition $\Omega \in H^{1}\left(S^{n-1}\right)$, where $H^{1}\left(S^{n-1}\right)$ is the Hardy space that contains $L \log ^{+} L\left(S^{n-1}\right)$ as a proper subspace on the unit sphere. Thus, a natural question is if one can use a weaker condition in Theorem B. The main purpose of this paper is to establish the following theorem.

THEOREM 1. If $\Omega \in H^{1}\left(S^{n-1}\right)$ and satisfies (1.1), then the operator $T$ is bounded on $L^{p}\left(\mathbb{R}^{n}\right)$ for $1<p<\infty$.

Before proving the theorem, we want to say a few words. First, we understand that the underlying space is a special homogeneous group (see [8]). Thus, many standard results might be adapted. For instance, we can use the Littlewood-Paley theory without any modifications. Second, although we follow the ideas in [7], we find that it is not an easy process of copy and paste. We must obtain some nontrivial estimates in our proof.

The reader can find these new estimates in Section 3. In Section 2 we present some basic definitions and known lemmas. The letter $C$ in the paper denotes positive constant independent of essential variables. 


\section{Definitions and lemmas}

The Poisson kernel on $S^{n-1}$ is defined by $P_{\mathrm{t} y^{\prime}}\left(x^{\prime}\right)=\left(1-\mathrm{t}^{2}\right) /\left|\mathrm{t} y^{\prime}-x^{\prime}\right|^{n}$ with $0 \leq \mathrm{t}<1, x^{\prime}, y^{\prime} \in S^{n-1}$. For any $\Omega \in L^{1}\left(S^{n-1}\right)$, we define the radial maximal function

$$
P^{+} \Omega\left(x^{\prime}\right)=\sup _{0 \leq \mathrm{t}<1}\left|\int_{S^{n-1}} \Omega\left(y^{\prime}\right) P_{\mathrm{t} x^{\prime}}\left(y^{\prime}\right) d \sigma\left(y^{\prime}\right)\right| .
$$

The Hardy space $H^{1}\left(S^{n-1}\right)$, is a subspace of $L^{1}\left(S^{n-1}\right)$ which contains all $L^{1}$ functions $\Omega$ with the finite norms $\|\Omega\|_{H^{1}\left(S^{n-1}\right)}=\left\|P^{+} \Omega\right\|_{L^{1}\left(S^{n-1}\right)}<\infty$.

An important property of $H^{1}\left(S^{n-1}\right)$ is the atomic decomposition, which is reviewed in the following. An exceptional atom $E\left(x^{\prime}\right)$ is an $L^{\infty}\left(S^{n-1}\right)$ function bounded by 1 . A regular $H^{1}\left(S^{n-1}\right)$ atom is an $L^{\infty}\left(S^{n-1}\right)$ function $a\left(x^{\prime}\right)$ satisfying the following conditions:

$$
\begin{gathered}
\operatorname{supp}(a) \subset S^{n-1} \cap\left\{y \in \mathbb{R}^{n}:\left|y-\xi^{\prime}\right|<r \text { for some } \xi^{\prime} \in S^{n-1} \text { and } r \in(0,1]\right\} \\
\int_{S^{n-1}} a\left(x^{\prime}\right) Y_{m}\left(x^{\prime}\right) d \sigma\left(x^{\prime}\right)=0
\end{gathered}
$$

for any spherical harmonic polynomial $Y_{m}$ with degree $m \leq N$, where $N$ is any fixed integer;

$$
\|a\|_{L^{\infty}\left(S^{n-1}\right)} \leq r^{1-n}
$$

From [3], we find that any $\Omega \in H^{1}\left(S^{n-1}\right)$ has an atomic decomposition

$$
\Omega=\sum_{j=1}^{\infty} \lambda_{j} a_{j}+\sum_{i=1}^{\infty} u_{i} E_{i}
$$

where each $a_{j}$ is a regular $H^{1}\left(S^{n-1}\right)$ atom and each $E_{i}$ is an exceptional atom. Moreover,

$$
\sum_{j=1}^{\infty}\left|\lambda_{j}\right|+\sum_{i=1}^{\infty}\left|u_{i}\right| \leq C\|\Omega\|_{H^{1}\left(S^{n-1}\right)} .
$$

We note that for any $x^{\prime} \in S^{n-1}$,

$$
\left|\sum_{i=1}^{\infty} u_{i} E_{i}\left(x^{\prime}\right)\right| \leq \sum_{i=1}^{\infty}\left|u_{i}\right|
$$

Without loss of generality, we can assume

$$
\left|\sum_{i=1}^{\infty} u_{i} E_{i}\left(x^{\prime}\right)\right| \leq\|\Omega\|_{H^{1}\left(S^{n-1}\right)} .
$$


Thus, we write

$$
\sum_{i=1}^{\infty} u_{i} E_{i}\left(x^{\prime}\right)=\|\Omega\|_{H^{1}\left(S^{n-1}\right)} \omega\left(x^{\prime}\right)
$$

with

$$
\omega\left(x^{\prime}\right)=\sum_{i=1}^{\infty} u_{i} E_{i}\left(x^{\prime}\right) /\|\Omega\|_{H^{1}\left(S^{n-1}\right)} .
$$

In this new definition,

$$
\Omega=\sum_{j=1}^{\infty} \lambda_{j} a_{j}+\|\Omega\|_{H^{1}\left(S^{n-1}\right)} \omega \quad \text { and } \quad\|\omega\|_{L^{\infty}\left(S^{n-1}\right)} \leq 1 .
$$

Noting that $J(x /|x|)|x|^{2}$ is a homogeneous polynomial of degree 2, by [11, Theorem 2.1], we can write $J(x /|x|)|x|^{2}=P_{2}(x)+|x|^{2} P_{0}(x)$, where $P_{k}(x)$ is a harmonic polynomial of degree $k(k=0,2)$. Then $J\left(x^{\prime}\right)=P_{2}\left(x^{\prime}\right)+P_{0}\left(x^{\prime}\right)$, where $P_{k}\left(x^{\prime}\right)$ is a spherical harmonic polynomial of degree $k(k=0,2)$. So by $(2.2)$, we have

$$
\begin{aligned}
\int_{S^{n-1}} a_{j}\left(y^{\prime}\right) J\left(y^{\prime}\right) d \sigma\left(y^{\prime}\right)= & \int_{S^{n-1}} a_{j}\left(y^{\prime}\right) P_{2}\left(y^{\prime}\right) d \sigma\left(y^{\prime}\right) \\
& +\int_{S^{n-1}} a_{j}\left(y^{\prime}\right) P_{0}\left(y^{\prime}\right) d \sigma\left(y^{\prime}\right)=0,
\end{aligned}
$$

for all $j=1,2, \ldots$ Thus, if $\Omega$ satisfies the cancellation condition (1.1), then

$$
\int_{S^{n-1}} \omega\left(y^{\prime}\right) J\left(y^{\prime}\right) d \sigma\left(y^{\prime}\right)=0 .
$$

The following Lemmas 2.1 and 2.2 can be found in [7].

LEMMA 2.1. Suppose that $n \geq 3$ and $b$ satisfies (2.1), (2.3) and

$$
\int_{S^{n-1}} b\left(y^{\prime}\right) d \sigma\left(y^{\prime}\right)=0 .
$$

Let

$$
F_{b}(s)=\left(1-s^{2}\right)^{(n-3) / 2} \chi_{(-1,1)}(s) \int_{S^{n-2}} b\left(s,\left(1-s^{2}\right)^{1 / 2} \tilde{y}\right) d \sigma(\tilde{y}),
$$

and

$$
G_{b}(s)=\left(1-s^{2}\right)^{(n-3) / 2} \chi_{(-1,1)}(s) \int_{S^{n-2}}\left|b\left(s,\left(1-s^{2}\right)^{1 / 2} \tilde{y}\right)\right| d \sigma(\tilde{y}) .
$$

Then there exists a constant $C$, independent of $b$, such that

$$
\begin{gathered}
\operatorname{supp}\left(F_{b}\right) \subset\left(\xi_{1}^{\prime}-2 r\left(\xi^{\prime}\right), \xi_{1}^{\prime}+2 r\left(\xi^{\prime}\right)\right) ; \\
\operatorname{supp}\left(G_{b}\right) \subset\left(\xi_{1}^{\prime}-2 r\left(\xi^{\prime}\right), \xi_{1}^{\prime}+2 r\left(\xi^{\prime}\right)\right) ; \\
\left\|F_{b}\right\|_{\infty} \leq C / r\left(\xi^{\prime}\right) ; \quad\left\|G_{b}\right\|_{\infty} \leq C / r\left(\xi^{\prime}\right) ; \\
\int_{\mathbb{R}} F_{b}(s) d s=0,
\end{gathered}
$$


where $r\left(\xi^{\prime}\right)=|\xi|^{-1}\left|L_{r} \xi\right|$ and $L_{r} \xi=\left(r^{2} \xi_{1}, r \xi_{2}, \ldots, r \xi_{n}\right)$.

Lemma 2.2. Suppose that $n=2$ and b satisfies (2.1), (2.3) and (2.6). Let

$$
F_{b}(s)=\left(1-s^{2}\right)^{-1 / 2} \chi_{(-1,1)}(s)\left(b\left(s,\left(1-s^{2}\right)^{1 / 2}\right)+b\left(s,-\left(1-s^{2}\right)^{1 / 2}\right)\right),
$$

and

$$
G_{b}(s)=\left(1-s^{2}\right)^{-1 / 2} \chi_{(-1,1)}(s)\left(\left|b\left(s,\left(1-s^{2}\right)^{1 / 2}\right)\right|+\left|b\left(s,-\left(1-s^{2}\right)^{1 / 2}\right)\right|\right) .
$$

Then $F_{b}(s)$ satisfies (2.7), (2.10) and

$$
\left\|F_{b}\right\|_{q} \leq C\left|L_{r}\left(\xi^{\prime}\right)\right|^{-1+1 / q},
$$

and $G_{b}(s)$ satisfies (2.8) and

$$
\left\|G_{b}\right\|_{q} \leq C\left|L_{r}\left(\xi^{\prime}\right)\right|^{-1+1 / q},
$$

for some $q \in(1,2)$.

LEMMA 2.3. Fix any function $\psi \in \mathcal{S}\left(\mathbb{R}^{n}\right)$ with $\operatorname{supp}(\psi) \subset\{x: 1 / 2 \leq \rho(x) \leq 2\}$. Let $\widehat{\Psi}(\xi)=\psi(\rho(\xi)), \Psi_{t}(\xi)=t^{-\alpha} \Psi\left(A_{t^{-1}} \xi\right)$ for $t>0$. For $j \in \mathbb{Z}$, define the multiplier $S_{j}$ by $\widehat{S_{j} f}(\xi)=\psi\left(2^{j} \rho(\xi)\right) \widehat{f}(\xi)$. Then for $1<p<\infty$, we have

$$
\left\|\left(\sum_{j}\left|S_{j} f\right|^{2}\right)^{1 / 2}\right\|_{p} \leq C\|f\|_{p}
$$

where $C$ is a constant independent of $f \in L^{p}\left(\mathbb{R}^{n}\right)$.

Lemma 2.3 is a discrete version of a more general theorem in [8]. One can prove Lemma 2.3 easily following the idea in [8].

Next, we let $\Phi(t)=e^{-\pi t^{2}}(t \in \mathbb{R})$. Then $\widehat{\Phi}(t)=e^{-\pi t^{2}}$. Define a radial function $\Phi_{k}$ on $\mathbb{R}^{n}$ by $\widehat{\Phi_{k}}(\xi)=\widehat{\Phi}\left(\left|L_{r} A_{2^{k}} \xi\right|\right)$. We have the following lemma.

LEMMA 2.4. The maximal operator $f \rightarrow \sup _{k}\left|\Phi_{k} * f\right|$ is bounded on $L^{p}\left(\mathbb{R}^{n}\right)$ for $1<p<\infty$.

PROOF. It is easy to check that

$$
\Phi_{k}(x)=r^{-2} 2^{-k \alpha_{1}} \Phi\left(x_{1} r^{-2} 2^{-k \alpha_{1}}\right) \prod_{j=2}^{n}\left\{r^{-1} 2^{-k \alpha_{j}} \Phi\left(x_{j} r^{-1} 2^{-k \alpha_{j}}\right)\right\} .
$$

Thus

$$
\sup _{k}\left|\Phi_{k} * f(x)\right| \leq C M_{1} M_{2} \cdots M_{n}(f)(x),
$$

where $M_{j}$ is the one-dimensional Hardy-Littlewood maximal operator acting on $x_{j}$ variable. So the lemma follows easily by the $L^{p}$ boundedness of the Hardy-Littlewood maximal function.

The following lemma is a variation of a lemma in [5]. 
LemMa 2.5. Let $r>0$. Suppose that there exist positive numbers $\gamma$ and $\beta$ such that

$$
\left|\widehat{\sigma_{k}}(\xi)\right| \leq C \min \left\{\left|L_{r} A_{2^{k}} \xi\right|^{-\beta},\left|L_{r} A_{2^{k}} \xi\right|^{\gamma}\right\}
$$

where $L_{r} A_{2^{k}} \xi=\left(r^{2} 2^{k \alpha_{1}} \xi_{1}, r 2^{k \alpha_{2}} \xi_{2}, \ldots, r 2^{k \alpha_{n}} \xi_{n}\right)$. Moreover, suppose also that, for some $q>1$,

$$
\left\|\sigma^{*}(f)\right\|_{q}=\left\|\sup _{k}|| \sigma_{k}|* f|\right\|_{q} \leq C\|f\|_{q},
$$

where $C>0$ is independent of $k \in \mathbb{Z}, \xi$ and $r$. Then the following two operators are bounded on $L^{p}\left(\mathbb{R}^{n}\right)$ uniformly for $r>0$, whenever $|1 / p-1 / 2|<1 / 2 q$ :

$$
B(f)=\sum_{k} \sigma_{k} * f, \quad g(f)=\left(\sum_{k}\left|\sigma_{k} * f\right|^{2}\right)^{1 / 2} .
$$

PROOF. If (2.12) holds and $1 / 2 q=\left|1 / 2-1 / p_{0}\right|$, then by [5], for arbitrary vector $\left\{h_{k}\right\}$ in $L^{p_{0}}\left(l^{2}\right)$, the following vector valued inequality holds

$$
\left\|\left(\sum_{k}\left|\sigma_{k} * h_{k}\right|^{2}\right)^{1 / 2}\right\|_{p_{0}} \leq C\left\|\left(\sum_{k}\left|h_{k}\right|^{2}\right)^{1 / 2}\right\|_{p_{0}} .
$$

Choose a $C_{0}^{\infty}$ function $\psi$ such that $0 \leq \psi \leq 1$ and $\operatorname{supp}(\psi) \subset\{y: 1 / 2 \leq \rho(y) \leq 2\}$ and $\sum_{j} \psi\left(2^{j} \rho\left(L_{r} \xi\right)\right)^{2}=1$. Define $\Upsilon$ and $\Delta$ by $\widehat{\Upsilon}(\xi)=\psi\left(\rho\left(L_{r} \xi\right)\right)$ and $\widehat{\Delta}(\xi)$ $=\psi(\rho(\xi))$. Denote $\Upsilon_{j}(x)=2^{-j \alpha} \Upsilon\left(A_{2^{-j}} x\right)$ and $\Delta_{j}(x)=2^{-j \alpha} \Delta\left(A_{2^{-j}} x\right)$ for $j \in \mathbb{Z}$. Then it is easy to check $\widehat{\Upsilon}_{j}(\xi)=\psi\left(2^{j} \rho\left(L_{r} \xi\right)\right)$ and $\widehat{\Delta_{j}}(\xi)=\psi\left(2^{j} \rho(\xi)\right)$ and $\Upsilon_{j}(x)=\left(1 / r^{n+1}\right) 2^{-j \alpha} \Delta\left(L_{1 / r} A_{2^{-j}} x\right)$. Define the multiplier $S_{j}$ on $\mathbb{R}^{n}$ by $\widehat{\left(S_{j} f\right)}(\xi)$ $=\psi\left(2^{j} \rho\left(L_{r} \xi\right)\right) \widehat{f}(\xi)$. Then we know $S_{j} f(x)=\Upsilon_{j} * f(x)$. Now we claim that

$$
\left\|\left(\sum_{j}\left|S_{j} f\right|^{2}\right)^{1 / 2}\right\|_{p} \leq C\|f\|_{p} \quad \text { for all } 1<p<\infty,
$$

with $C$ independent of $r$. In fact, by the definition of $\Upsilon_{j}$, we have

$$
\begin{aligned}
\Upsilon_{j} * f(x) & =\frac{1}{r^{n+1}} 2^{-j \alpha} \int_{\mathbb{R}^{n}} \Delta\left(L_{1 / r} A_{2^{-j}} y\right) f(x-y) d y \\
& =2^{-j \alpha} \int_{\mathbb{R}^{n}} \Delta\left(A_{2^{-j}} y\right) f\left(L_{r}\left(L_{1 / r} x-y\right)\right) d y \\
& =\Delta_{j} * U\left(L_{1 / r} x\right),
\end{aligned}
$$

where $U(x)=f\left(L_{r} x\right)$. Then, by Lemma 2.3, we obtain 


$$
\begin{aligned}
\left\|\left(\sum_{j}\left|S_{j} f(x)\right|^{2}\right)^{1 / 2}\right\|_{p} & =\left\{\int_{\mathbb{R}^{n}}\left(\sum_{j}\left|\Delta_{j} * U\left(L_{1 / r} x\right)\right|^{2}\right)^{p / 2} d x\right\}^{1 / p} \\
& =\left\{r^{n+1} \int_{\mathbb{R}^{n}}\left(\sum_{j}\left|\Delta_{j} * U(x)\right|^{2}\right)^{p / 2} d x\right\}^{1 / p} \\
& \leq C r^{(n+1) / p}\|U\|_{p} \\
& =C\left(r^{n+1} \int_{\mathbb{R}^{n}}\left|f\left(L_{r} x\right)\right|^{p} d x\right)^{1 / p}=C\|f\|_{p} .
\end{aligned}
$$

Thus, (2.14) is proved. By the definition of $S_{j}$, we have, for any $f \in \mathcal{S}\left(\mathbb{R}^{n}\right)$, $\sum_{j} S_{j}^{2} f(x)=\sum_{j} S_{j}\left(S_{j} f\right)(x)=f(x)$. We decompose operator $B$ as follows:

$$
B(f)=\sum_{k} \sigma_{k} *\left(\sum_{j} S_{j+k} S_{j+k} f\right)=\sum_{j} \sum_{k}\left(S_{j+k}\left(\sigma_{k} * S_{j+k} f\right)\right)=\sum_{j} \mathcal{B}_{j}(f) .
$$

First we estimate $\mathcal{B}_{j}$ in $L^{p_{0}}$. By (2.13) and (2.14), we have

$$
\begin{aligned}
\left\|\mathcal{B}_{j}(f)\right\|_{p_{0}} & \leq C\left\|\left(\sum_{k}\left|\sigma_{k} * S_{j+k} f\right|^{2}\right)^{1 / 2}\right\|_{p_{0}} \\
& \leq C\left\|\left(\sum_{k}\left|S_{j+k} f\right|^{2}\right)^{1 / 2}\right\|_{p_{0}} \\
& \leq C\|f\|_{p_{0}} .
\end{aligned}
$$

Now we compute the $L^{2}$-norm of $\mathcal{B}_{j}(f)$. When $j<0$, by using the estimate $\left|\widehat{\sigma_{k}}(\xi)\right| \leq C\left|L_{r} A_{2^{k}} \xi\right|^{-\beta}$ we have

$$
\begin{aligned}
& \left\|\mathcal{B}_{j}(f)\right\|_{2}^{2} \leq \sum_{k} \int_{2^{-j-k-1} \leq \rho\left(L_{r} \xi\right) \leq 2^{-j-k+1}}|\widehat{f}(\xi)|^{2}\left|L_{r} A_{2^{k}} \xi\right|^{-2 \beta} d \xi \\
& \quad=\frac{1}{r^{n+1}} \sum_{k} \int_{2^{-j-k-1}}^{2^{-j-k+1}} \int_{S^{n-1}} J\left(\xi^{\prime}\right)\left|\widehat{f}\left(L_{r^{-1}} A_{\rho} \xi^{\prime}\right)\right|^{2}\left|A_{2^{k}} A_{\rho} \xi^{\prime}\right|^{-2 \beta} \rho^{\alpha-1} d \sigma\left(\xi^{\prime}\right) d \rho \\
& \quad \leq C 2^{2 \beta j \alpha_{1}} \frac{1}{r^{n+1}} \sum_{k} \int_{2^{-j-k-1}}^{2^{-j-k+1}} \int_{S^{n-1}} J\left(\xi^{\prime}\right)\left|\widehat{f}\left(L_{r^{-1}} A_{\rho} \xi^{\prime}\right)\right|^{2} \rho^{\alpha-1} d \sigma\left(\xi^{\prime}\right) d \rho \\
& \quad \leq C 2^{2 \beta j} \frac{1}{r^{n+1}} \int_{\mathbb{R}^{n}}\left|\widehat{f}\left(L_{r^{-1}} \xi\right)\right|^{2} d \xi \\
& \quad=C 2^{2 \beta j}\|f\|_{2}^{2} .
\end{aligned}
$$

So we obtain

$$
\left\|\mathcal{B}_{j}(f)\right\|_{2} \leq C 2^{\beta j}\|f\|_{2} \text { for all } j<0
$$


If $j>0$, using the estimate $\left|\widehat{\sigma}_{k}(\xi)\right| \leq\left|L_{r} A_{2^{k}} \xi\right|^{\gamma}$ and the same idea of proving (2.16), we have

$$
\left\|\mathcal{B}_{j}(f)\right\|_{2} \leq C 2^{-\gamma j}\|f\|_{2} \text { for all } j>0,
$$

where $C$ is independent of $j$ and $f$. Thus, by (2.16) and (2.17) we obtain

$$
\left\|\mathcal{B}_{j}(f)\right\|_{2} \leq C 2^{-\delta|j|}\|f\|_{2} \quad \text { for all } \delta>0 .
$$

Now, if $|1 / p-1 / 2|<1 / 2 q$, we have $1 / p=\theta / 2+(1-\theta) / p_{0}$ for some $0<\theta<1$. By interpolating between (2.15) and (2.18), we obtain

$$
\|B(f)\|_{p} \leq \sum_{j}\left\|\mathcal{B}_{j}(f)\right\|_{p} \leq C \sum_{j} 2^{-\delta \kappa|j|}\|f\|_{p} \leq C\|f\|_{p} \quad \text { for all } 0<\kappa<1 .
$$

The inequality $\|g(f)\|_{p} \leq C\|f\|_{p}$ can be proved by essentially the same argument.

LEMmA 2.6 (See [5]). Let $\left\{\eta_{k}\right\}$ be a lacunary sequence of positive numbers $\left.\inf _{k}\left(\eta_{k+1} / \eta_{k}\right)=\eta>1\right)$. Suppose that $\left\{\lambda_{k}\right\}$ is a sequence of nonnegative functions satisfying, for some $\theta>0$,

$$
\left|\widehat{\lambda_{k}}(\xi)-1\right| \leq C\left|\eta_{k+1} \xi\right|^{\theta}, \quad\left|\widehat{\lambda_{k}}(\xi)\right| \leq C\left|\eta_{k} \xi\right|^{-\theta},
$$

for all $k \in \mathbb{Z}$. Then, the maximal operator $\left(\lambda^{*} f\right)(x)=\sup _{k}\left|\lambda_{k} * f(x)\right|$ is bounded on $L^{p}\left(\mathbb{R}^{n}\right)$ for $1<p \leq \infty$.

Lemma 2.7 (See [4]). Suppose that $m$ denotes the distinct numbers of $\left\{\alpha_{j}\right\}$. Then for any $x, y \in \mathbb{R}^{n}, 0 \leq \beta \leq 1$

$$
\left|\int_{1}^{2} e^{-i A_{\lambda} x \cdot y} \frac{d \lambda}{\lambda}\right| \leq C|x \cdot y|^{-(\beta / m)}
$$

where $C>0$ is independent of $x$ and $y$.

\section{Proof of Theorem 1}

Noting that

$$
T f(x)=\int_{0}^{\infty} \int_{S^{n-1}} \Omega\left(y^{\prime}\right) J\left(y^{\prime}\right) f\left(x-A_{\rho} y^{\prime}\right) d \sigma\left(y^{\prime}\right) \frac{d \rho}{\rho} .
$$

Since $\Omega \in H^{1}\left(S^{n-1}\right)$ satisfying the cancellation condition (1.1), we can write

$$
\Omega=\sum_{j=1}^{\infty} \lambda_{j} a_{j}+\|\Omega\|_{H^{1}\left(S^{n-1}\right)} \omega,
$$


where $\omega$ satisfies (2.5) and $\|\omega\|_{L^{\infty}\left(S^{n-1}\right)} \leq 1$, each $a_{j}$ is a regular $H^{1}\left(S^{n-1}\right)$ atom and $\sum_{j=1}^{\infty}\left|\lambda_{j}\right| \leq C\|\Omega\|_{H^{1}\left(S^{n-1}\right)}$. So

$$
\|T f\|_{p} \leq C \sum_{j=1}^{\infty}\left|\lambda_{j}\right|\left\|B_{j}(f)\right\|_{p}+\|\Omega\|_{H^{1}\left(S^{n-1}\right)}\left\|T_{\omega} f\right\|_{p},
$$

where

$$
B_{j}(f)(x)=\int_{0}^{\infty} \int_{S^{n-1}} a_{j}\left(y^{\prime}\right) J\left(y^{\prime}\right) f\left(x-A_{\rho} y^{\prime}\right) d \sigma\left(y^{\prime}\right) \frac{d \rho}{\rho},
$$

and

$$
T_{\omega}(f)(x)=\int_{\mathbb{R}^{n}} f(x-y) \omega\left(y^{\prime}\right) \rho(y)^{-\alpha} d y .
$$

Since $\omega\left(y^{\prime}\right) \in L^{\infty}\left(S^{n-1}\right) \subset L \log ^{+} L\left(S^{n-1}\right)$ and satisfies (2.5), by Theorem B, we obtain

$$
\left\|T_{\omega} f\right\|_{p} \leq C\|f\|_{p},
$$

where $C$ is independent of $\omega$ and $f$. Therefore, to prove Theorem 1, it suffices by (3.1) and (3.2) to show that

$$
\left\|B_{j}(f)\right\|_{p} \leq C\|f\|_{p}, \quad j=1,2, \ldots,
$$

where $C$ is independent of the atoms $a_{j}$ and $f$. By (2.4) and by the observation that $J\left(y^{\prime}\right) \in C_{0}^{\infty}\left(S^{n-1}\right)$, it is easy to check that $\widetilde{a}_{j}\left(y^{\prime}\right)=a_{j}\left(y^{\prime}\right) J\left(y^{\prime}\right) /\|J\|_{L^{\infty}\left(S^{n-1}\right)}$ satisfies (2.1), (2.3) and (2.6). Thus,

$$
B_{j}(f)(x)=\|J\|_{L^{\infty}\left(S^{n-1}\right)} \int_{0}^{\infty} \int_{S^{n-1}} \tilde{a}_{j}\left(y^{\prime}\right) f\left(x-A_{\rho} y^{\prime}\right) d \sigma\left(y^{\prime}\right) \frac{d \rho}{\rho} .
$$

For simplicity in our argument, we denote $\tilde{a}_{j}$ by $a$ and $B_{j}(f) /\|J\|_{L^{\infty}\left(S^{n-1}\right)}$ by $B(f)$ from now. Without loss of generality, we may assume that $\operatorname{supp}(a) \subset B(\iota, r) \cap S^{n-1}$, where $\iota=(1,0, \ldots, 0)$ and $B(\iota, r)=\{y:|y-\iota|<r\}$. Let $I_{k}=\left(2^{k}, 2^{k+1}\right)$. Then $B(f)(x)$ is equal to

$$
\int_{0}^{\infty} \int_{S^{n-1}} \rho^{-1} a\left(y^{\prime}\right) \sum_{k} \chi_{I_{k}}(\rho) f\left(x-A_{\rho} y^{\prime}\right) d \sigma\left(y^{\prime}\right) d \rho=\sum_{k} \sigma_{k} * f(x),
$$

where

$$
\widehat{\sigma_{k}}(\xi)=\int_{I_{k}} \int_{S^{n-1}} a\left(y^{\prime}\right) e^{-2 \pi i A_{\rho} y^{\prime} \cdot \xi} d \sigma\left(y^{\prime}\right) \frac{d \rho}{\rho} .
$$

Let

$$
\widehat{\mu_{k}}(\xi)=\int_{I_{k}} \int_{S^{n-1}}\left|a\left(y^{\prime}\right)\right| e^{-2 \pi i A_{\rho} y^{\prime} \cdot \xi} d \sigma\left(y^{\prime}\right) \frac{d \rho}{\rho},
$$

and 


$$
\sigma^{*}(f)(x)=\sup _{k}\left|\mu_{k} * f(x)\right| .
$$

Then it is easy to verify that $\left\|\widehat{\mu_{k}}\right\|_{\infty}<C,\left\|\sigma_{k}\right\|_{1}<C$ uniformly for $k \in \mathbb{Z}$ and for all $k \in \mathbb{Z}$. Since $a\left(y^{\prime}\right)$ satisfies (2.6), then

$$
\widehat{\sigma_{k}}(0)=\int_{2^{k}}^{2^{k+1}} \frac{d \rho}{\rho} \int_{S^{n-1}} a\left(y^{\prime}\right) d \sigma\left(y^{\prime}\right)=0 .
$$

By Lemma 2.5, if we can show that $\left\{\sigma_{k}\right\}$ satisfies condition (2.11) and

$$
\left\|\sigma^{*}(f)\right\|_{p} \leq C\|f\|_{p} \text { for all } 1<p<\infty,
$$

where $C$ is independent of $a$ and $f$. Thus (3.3) is obtained. We first verify that $\left\{\sigma_{k}\right\}$ satisfies (2.11). We only prove the case $n>2$, since the proof for $n=2$ is essentially the same (using Lemma 2.2 instead of Lemma 2.1). For any $\xi \neq 0$, we choose a rotation $\mathcal{O}$ such that $\mathcal{O}\left(A_{\rho} \xi\right)=\left|A_{\rho} \xi\right| \iota=\left|A_{\rho} \xi\right|(1,0, \ldots, 0)$. Let $y^{\prime}=\left(s, y_{2}^{\prime}, y_{3}^{\prime}, \ldots, y_{n}^{\prime}\right)$. Then it is easy to see that

$$
\widehat{\sigma_{k}}(\xi)=\int_{I_{k}} \int_{S^{n-1}} a\left(\mathcal{O}^{-1}\left(y^{\prime}\right)\right) e^{-2 \pi i\left|A_{\rho} \xi\right|\left(\iota \cdot y^{\prime}\right)} d \sigma\left(y^{\prime}\right) \frac{d \rho}{\rho},
$$

where $\mathcal{O}^{-1}$ is the inverse of $\mathcal{O}$. Now $a\left(\mathcal{O}^{-1}\left(y^{\prime}\right)\right)$ also satisfies (2.3), (2.6) and is supported in $B(\zeta, r) \cap S^{n-1}$, where $\zeta=A_{\rho} \xi /\left|A_{\rho} \xi\right|$. Thus,

$$
\widehat{\sigma_{k}}(\xi)=\int_{I_{k}} \int_{\mathbb{R}} F_{a}(s) e^{-2 \pi i\left|A_{\rho} \xi\right| s} d s \frac{d \rho}{\rho},
$$

where $F_{a}(s)$ is the function defined in Lemma 2.1. By Lemma 2.1, we know $\operatorname{supp}\left(F_{a}\right) \subset\left(-2 r(\zeta)+\zeta_{1}, 2 r(\zeta)+\zeta_{1}\right)$, where $r(\zeta)=\left|L_{r} A_{\rho} \xi\right| /\left|A_{\rho} \xi\right|$ and $\zeta_{1}=\rho^{\alpha_{1}} \xi_{1} /\left|A_{\rho} \xi\right|$. Thus, $N(s)=r(\zeta) F_{a}(r(\zeta) s)$ is a function supported in the interval $\left(-2+\zeta_{1} / r(\zeta), 2+\zeta_{1} / r(\zeta)\right)$, and $\|N\|_{\infty}<C(C$ is independent of $s$ and $\rho)$, $\int_{\mathbb{R}} N(s) d s=0$. After changing variables

$$
\widehat{\sigma_{k}}(\xi)=\int_{I_{k}} \int_{\mathbb{R}} N(s) e^{-2 \pi i s\left|L_{r} A_{\rho} \xi\right|} d s \frac{d \rho}{\rho} .
$$

So by the cancellation property of $N(\cdot)$, we obtain that

$$
\begin{aligned}
\left|\widehat{\sigma_{k}}(\xi)\right| & \leq \int_{I_{k}}\left|\int_{\mathbb{R}} N(s)\left(e^{-2 \pi i\left|L_{r} A_{\rho} \xi\right| s}-e^{-2 \pi i \rho^{\alpha} \xi_{1}}\right) d s\right| \frac{d \rho}{\rho} \\
& \leq C \int_{I_{k}} \int_{\left|s-\frac{\zeta_{1}}{r(\zeta)}\right| \leq 2}|N(s)|\left|L_{r} A_{\rho} \xi\right|\left|s-\frac{\zeta_{1}}{r(\zeta)}\right| d s \frac{d \rho}{\rho} \\
& \leq C \int_{1}^{2}\left|L_{r} A_{2^{k}} \xi\right| \frac{d \rho}{\rho} \\
& \leq C\left|L_{r} A_{2^{k}} \xi\right| .
\end{aligned}
$$


On the other hand, by (3.4) and Hölder's inequality,

$$
\left|\widehat{\sigma_{k}(\xi)}\right|^{2} \leq C H_{k}(\xi),
$$

where

$$
H_{k}(\xi)=\int_{I_{k}}\left|\int_{S^{n-1}} a\left(y^{\prime}\right) e^{-2 \pi i A_{\rho} y^{\prime} \cdot \xi} d \sigma\left(y^{\prime}\right)\right|^{2} \frac{d \rho}{\rho} .
$$

Then we obtain

$$
\begin{aligned}
H_{k}(\xi) & =\int_{2^{k}}^{2^{k+1}} \iint_{S^{n-1} \times S^{n-1}} a\left(y^{\prime}\right) \overline{a\left(x^{\prime}\right)} e^{-2 \pi i A_{\rho}\left(y^{\prime}-x^{\prime}\right) \cdot \xi} d \sigma\left(y^{\prime}\right) d \sigma\left(x^{\prime}\right) \frac{d \rho}{\rho} \\
& \leq C \iint_{S^{n-1} \times S^{n-1}}\left|a\left(y^{\prime}\right)\right|\left|a\left(x^{\prime}\right)\right|\left|\int_{2^{k}}^{2^{k+1}} e^{-2 \pi i A_{\rho}\left(y^{\prime}-x^{\prime}\right) \cdot \xi} \frac{d \rho}{\rho}\right| d \sigma\left(y^{\prime}\right) d \sigma\left(x^{\prime}\right) .
\end{aligned}
$$

By Lemma 2.7, we know

$$
\begin{aligned}
\left|\int_{2^{k}}^{2^{k+1}} e^{-2 \pi i A_{\rho}\left(y^{\prime}-x^{\prime}\right) \cdot \xi} \frac{d \rho}{\rho}\right| & =\left|\int_{1}^{2} e^{-2 \pi i A_{2^{k}}\left(y^{\prime}-x^{\prime}\right) \cdot \xi} \frac{d \rho}{\rho}\right| \\
& \leq C\left(\left|\left(y^{\prime}-x^{\prime}\right) \cdot A_{2^{k}} \xi\right|\right)^{-2 \beta / m} .
\end{aligned}
$$

where $0 \leq \beta<1 / 2$ and $m$ denotes the distinct numbers of $\left\{\alpha_{j}\right\}$. Then by the above inequality we obtain

$$
H_{k}(\xi) \leq C \iint_{S^{n-1} \times S^{n-1}}\left|a\left(y^{\prime}\right)\right|\left|a\left(x^{\prime}\right)\right|\left(\left|\left(y^{\prime}-x^{\prime}\right) \cdot A_{2^{k}} \xi\right|\right)^{-2 \beta / m} d \sigma\left(y^{\prime}\right) d \sigma\left(x^{\prime}\right) .
$$

Denote

$$
I_{1}(\xi)=\iint_{S^{n-1} \times S^{n-1}}\left|a\left(y^{\prime}\right)\right|\left|a\left(x^{\prime}\right)\right|\left(\left|\left(y^{\prime}-x^{\prime}\right) \cdot A_{2^{k}} \xi\right|\right)^{-2 \beta / m} d \sigma\left(y^{\prime}\right) d \sigma\left(x^{\prime}\right) .
$$

For any $\xi \neq 0$, we choose a rotation $\mathcal{O}$ such that

$$
\mathcal{O}\left(A_{2^{k}} \xi\right)=\left|A_{2^{k}} \xi\right| \iota=\left|A_{2^{k}} \xi\right|(1,0, \ldots, 0) .
$$

Let

$$
y^{\prime}=\left(s, y_{2}^{\prime}, y_{3}^{\prime}, \ldots, y_{n}^{\prime}\right) \text { and } x^{\prime}=\left(t, x_{2}^{\prime}, x_{3}^{\prime}, \ldots, x_{n}^{\prime}\right) .
$$

Then it is easy to see that

$$
\begin{aligned}
I_{1}(\xi)= & \iint_{S^{n-1} \times S^{n-1}}\left|a\left(\mathcal{O}^{-1}\left(y^{\prime}\right)\right)\right|\left|a\left(\mathcal{O}^{-1}\left(x^{\prime}\right)\right)\right| \\
& \times\left(\left|\left(y^{\prime}-x^{\prime}\right) \cdot\right| A_{2^{k}} \xi|\iota|\right)^{-2 \beta / m} d \sigma\left(y^{\prime}\right) d \sigma\left(x^{\prime}\right),
\end{aligned}
$$


where $\mathcal{O}^{-1}$ is the inverse of $\mathcal{O}$. Now $a\left(\mathcal{O}^{-1}\left(y^{\prime}\right)\right)$ also satisfies (2.3), (2.6) and is supported in $B(\vartheta, r) \cap S^{n-1}$, where $\vartheta=A_{2^{k}} \xi /\left|A_{2^{k}} \xi\right|$. Thus,

$$
I_{1}(\xi)=\iint_{\mathbb{R} \times \mathbb{R}} G_{a}(s) G_{a}(t)\left(\left|A_{2^{k}} \xi \| s-t\right|\right)^{-2 \beta / m} d s d t
$$

where $G_{a}(s)$ is the function defined in Lemma 2.1. By Lemma 2.1, we know $\operatorname{supp}\left(G_{a}\right) \subset\left(-2 r(\vartheta)+\vartheta_{1}, 2 r(\vartheta)+\vartheta_{1}\right)$, where $r(\vartheta)=\left|L_{r} A_{2^{k}} \xi\right| /\left|A_{2^{k}} \xi\right|$ and $\vartheta_{1}=2^{k^{\alpha_{1}}} \xi_{1} /\left|A_{2^{k}} \xi\right|$. Thus,

$$
\varphi(s)=r(\vartheta) G_{a}\left(r(\vartheta)\left(s-\frac{\vartheta_{1}}{r(\vartheta)}\right)\right)
$$

is a function supported in the interval $(-2,2)$, and $\|\varphi\|_{\infty}<C$, where $C$ is independent of $r, \vartheta$ and $k$. Since $2 \beta / m<1$, we obtain

$$
\begin{aligned}
I_{1}(\xi) & =\int_{-2}^{2} \int_{-2}^{2} \varphi(s) \varphi(t)\left(\left|L_{r} A_{2^{k}} \xi\right||s-t|\right)^{-2 \beta / m} d s d t \\
& \leq C\left|L_{r} A_{2^{k}} \xi\right|^{-2 \beta / m} \int_{-2}^{2} \int_{-2}^{2}|s-t|^{-2 \beta / m} d s d t \\
& \leq C\left|L_{r} A_{2^{k}} \xi\right|^{-2 \beta / m} .
\end{aligned}
$$

This together with (3.9) and (3.9') gives

$$
\left|\widehat{\sigma_{k}}(\xi)\right| \leq C\left|L_{r} A_{2^{k}} \xi\right|^{-\beta / m} .
$$

By (3.8) and (3.10),

$$
\left|\widehat{\sigma}_{k}(\xi)\right| \leq C \min \left\{\left|L_{r} A_{2^{k}} \xi\right|,\left|L_{r} A_{2^{k}} \xi\right|^{-\beta / m}\right\} .
$$

Inequality (3.11) shows that $\left\{\sigma_{k}\right\}$ satisfies (2.11). Hence, it remains to show (3.7). We define the measure sequences $\left\{\lambda_{k}\right\}$ on $\mathbb{R}$ by

$$
\widehat{\lambda_{k}}\left(\xi_{1}\right)=\|a\|_{L^{1}\left(S^{n-1}\right)} \int_{I_{k}} e^{-2 \pi i \rho^{\alpha} \xi_{1}} \frac{d \rho}{\rho} .
$$

Let $\delta$ be the Dirac delta function acting on $\left(x_{2}, \ldots, x_{n}\right)$. Now, choose the function $\Phi_{k}$ as in Lemma 2.4 and for each $k$ define

$$
v_{k}=\mu_{k}-\Phi_{k} *\left(\lambda_{k} \otimes \delta\right) .
$$

By (3.6) and (3.13),

$$
\sigma^{*}(f) \leq\left(\sum_{k}\left|v_{k} * f\right|^{2}\right)^{1 / 2}+\sup _{k} \Phi_{k} *\left(\sup _{k}\left|\left(\lambda_{k} \otimes \delta\right) * f\right|\right),
$$


and

$$
|| v_{k}|* f| \leq \sigma^{*}(f)+\sup _{k} \Phi_{k} *\left(\sup _{k}\left|\left(\lambda_{k} \otimes \delta\right) * f\right|\right) .
$$

By (3.14), if we can prove

$$
\left\|\sup _{k} \Phi_{k} *\left(\sup _{k}\left|\left(\lambda_{k} \otimes \delta\right) * f\right|\right)\right\|_{p} \leq C\|f\|_{p} \quad \text { for all } 1<p<\infty,
$$

and

$$
\left\|\left(\sum_{k}\left|v_{k} * f\right|^{2}\right)^{1 / 2}\right\|_{p} \leq C\|f\|_{p} \quad \text { for all } 1<p<\infty,
$$

where $C$ is independent of $f$, then we obtain (3.7). We first verify (3.16). By the definition of $\lambda_{k}$, it is easy to see that, for each $k,\left|\widehat{\lambda_{k}}\left(\xi_{1}\right)\right| \leq C$, and $\lambda_{k}$ is a positive measure on $\mathbb{R}$ :

$$
\begin{aligned}
\left|\widehat{\lambda_{k}}\left(\xi_{1}\right)-\widehat{\lambda_{k}}(0)\right| & \leq\|a\|_{L^{1}\left(S^{n-1}\right)} \int_{I_{k}}\left|e^{-2 \pi i \rho^{\alpha} 1 \xi_{1}}-1\right| \frac{d \rho}{\rho} \\
& \leq C 2^{k \alpha_{1}}\left|\xi_{1}\right| .
\end{aligned}
$$

Using integration by parts,

$$
\left|\widehat{\lambda_{k}}\left(\xi_{1}\right)\right| \leq C\left(2^{k \alpha_{1}}\left|\xi_{1}\right|\right)^{-1} .
$$

By Lemma 2.6, we know that $\sup _{k}\left|\lambda_{k} * f\right|$ is bounded in $L^{p}(\mathbb{R})$ for $1<p<\infty$. Since $\delta$ is the Dirac delta function acting on $\left(x_{2}, \ldots, x_{n}\right)$, we see $\sup _{k}\left|\left(\lambda_{k} \otimes \delta\right) * f\right|$ is bounded on $L^{p}\left(\mathbb{R}^{n}\right)$. So by Lemma 2.4 , we obtain (3.16). Hence, it remains to show (3.17). We still use Lemma 2.5 to do this. We first show that $\left\{v_{k}\right\}$ satisfies (2.11). By (3.13),

$$
\left|\widehat{v_{k}}(\xi)\right| \leq\left|\widehat{\mu_{k}}(\xi)-\widehat{\lambda_{k}}\left(\xi_{1}\right)\right|+\left|\widehat{\lambda_{k}}\left(\xi_{1}\right)\right|\left|\widehat{\Phi_{k}}(\xi)-1\right|,
$$

and

$$
\left|\widehat{v_{k}}(\xi)\right| \leq\left|\widehat{\mu_{k}}(\xi)\right|+\left|\widehat{\lambda_{k}}\left(\xi_{1}\right)\right|\left|\widehat{\Phi_{k}}(\xi)\right| .
$$

By (3.5) and applying the method of rotation again

$$
\widehat{\mu_{k}}(\xi)=C \int_{I_{k}} \int_{\mathbb{R}} G_{a}(s) e^{-2 \pi i\left|A_{\rho} \xi\right| s} d s \frac{d \rho}{\rho} .
$$

Note that $\operatorname{supp}\left(G_{a}\right)=\operatorname{supp}\left(F_{a}\right)=\left(\zeta_{1}-2 r(\zeta), \zeta_{1}+2 r(\zeta)\right)$ by (2.7) and (2.8), and also note $\int_{\mathbb{R}} G_{a}(s) d s=\|a\|_{L^{1}\left(S^{n-1}\right)}$, then by Lemma 2.1 we have

$$
\begin{aligned}
\left|\widehat{\mu_{k}}(\xi)-\widehat{\lambda_{k}}\left(\xi_{1}\right)\right| & \leq \int_{I_{k}} \int_{\mathbb{R}}\left|G_{a}(s)\right|\left|e^{-2 \pi i\left|A_{\rho} \xi\right| s}-e^{-2 \pi i \rho^{\alpha} 1 \xi_{1}}\right| d s \frac{d \rho}{\rho} \\
& \leq C \int_{I_{k}} \frac{1}{r(\zeta)} \int_{\left|s-\zeta_{1}\right| \leq 2 r(\zeta)}\left|A_{\rho} \xi\right|\left|s-\zeta_{1}\right| d s \frac{d \rho}{\rho} \\
& \leq C\left|L_{r} A_{2^{k}} \xi\right| .
\end{aligned}
$$


From the proof of (3.10), we also have

$$
\left|\widehat{\mu_{k}}(\xi)\right| \leq C\left|L_{r} A_{2^{k}} \xi\right|^{-\beta / m} .
$$

On the other hand,

$$
\widehat{\Phi_{k}}(\xi)=\widehat{\Phi}\left(\left|L_{r} A_{2^{k}} \xi\right|\right)=e^{-\pi\left|L_{r} A_{2^{k}} \xi\right|^{2}}=\int_{\mathbb{R}} e^{-\pi t^{2}} e^{-2 \pi i t\left|L_{r} A_{2^{k}} \xi\right|} d t .
$$

Thus,

$$
\begin{aligned}
\left|\widehat{\Phi_{k}}(\xi)-1\right| & =\left|\int_{\mathbb{R}} e^{-\pi t^{2}}\left(e^{-2 \pi i t\left|L_{r} A_{2^{k}} \xi\right|}-1\right) d t\right| \\
& \leq C\left|L_{r} A_{2^{k}} \xi\right| \int_{0}^{\infty} e^{-\pi t^{2}} t d t \leq C\left|L_{r} A_{2^{k}} \xi\right|
\end{aligned}
$$

Using integration by parts,

$$
\left|\widehat{\Phi_{k}}(\xi)\right| \leq \frac{C}{\left|L_{r} A_{2^{k}} \xi\right|} \int_{0}^{\infty} e^{-\pi t^{2}} t d t \leq C\left|L_{r} A_{2^{k}} \xi\right|^{-1}
$$

By (3.18), (3.20), (3.22) and $\left|\widehat{\lambda_{k}}\left(\xi_{1}\right)\right| \leq C$, we have

$$
\left|\widehat{v_{k}}(\xi)\right| \leq C\left|L_{r} A_{2^{k}} \xi\right| .
$$

On the other hand, by (3.19), (3.21), (3.23) and $\left|\widehat{\lambda_{k}}\left(\xi_{1}\right)\right| \leq C$, it is easy to see that

$$
\left|\widehat{v_{k}}(\xi)\right| \leq C\left|L_{r} A_{2^{k}} \xi\right|^{-\eta},
$$

where $\left|L_{r} A_{2^{k}} \xi\right|^{-\eta}=\max \left\{\left|L_{r} A_{2^{k}} \xi\right|^{-1},\left|L_{r} A_{2^{k}} \xi\right|^{-\beta / m}\right\}$ and $\eta=\beta / m$ or 1 . Thus, (3.24) and (3.25) yield

$$
\left|\widehat{v_{k}}(\xi)\right| \leq C \min \left\{\left|L_{r} A_{2^{k}} \xi\right|,\left|L_{r} A_{2^{k}} \xi\right|^{-\eta}\right\} .
$$

So $\left\{v_{k}\right\}$ satisfies (2.11). By (3.26) and the same idea of proving (2.18) in Lemma 2.5, we obtain

$$
\left\|\left(\sum_{k}\left|v_{k} * f\right|^{2}\right)^{1 / 2}\right\|_{2} \leq C\|f\|_{2},
$$

where $C$ is independent of $f$. By (3.14) and (3.16), we see $\left\|\sigma^{*}(f)\right\|_{2} \leq C\|f\|_{2}$. Therefore, by (3.15), (3.16), we obtain $\left\|\sup _{k}\right\| v_{k}|* f|\left\|_{2} \leq C\right\| f \|_{2}$. Applying Lemma 2.5 with $q=2$ again, we obtain

$$
\left\|\left(\sum_{k}\left|v_{k} * f\right|^{2}\right)^{1 / 2}\right\|_{p} \leq C\|f\|_{p} \quad \text { for all } 4 / 3<p<4 .
$$


and

$$
\left\|\sigma^{*}(f)\right\|_{p} \leq C\|f\|_{p} \quad \text { for all } 4 / 3<p<4 \text {. }
$$

Thus, a bootstrap argument by reiterating application of Lemma 2.5 gives us

$$
\left\|\left(\sum_{k}\left|v_{k} * f\right|^{2}\right)^{1 / 2}\right\|_{p} \leq C\|f\|_{p},
$$

for all $1<p<\infty$. Thus, (3.17) is proved and Theorem 1 follows.

\section{References}

[1] A. Calderón and A. Zygmund, 'On the existence of certain singular integrals', Acta Math. 88 (1952), 85-139.

[2] A. Calderón and A. Zygmund, 'Singular integral operators and differential equations', Amer. J. Math. 79 (1957), 901-921.

[3] L. Colzani, 'Hardy spaces on sphere', PhD Thesis, Washington University, St Louis, MO, 1982.

[4] Y. Ding, Q. Xue and K. Yabuta, 'Parabolic Littlewood-Paley g-function with rough kernels', Acta Math. Sin. (Engl. Ser.) 24 (2008), to appear.

[5] J. Duoandikoetxea and J. L. Rubio de Francia, 'Maximal and singular integral operators via Fourier transform estimates', Invent. Math. 84 (1986), 541-561.

[6] E. B. Fabes and N. M. Rivière, 'Singular integrals with mixed homogeneity', Studia Math. 27 (1966), 19-38.

[7] D. Fan and Y. Pan, 'A singular integral operator with rough kernel', Proc. Amer. Math. Soc. 125 (1997), 3695-3703.

[8] G. B. Folland and E. M. Stein, Hardy Spaces on Homogeneous Groups, Mathematical Notes, 28 (Princeton University Press, Princeton, NJ, 1982).

[9] A. Nagel, N. M. Riviere and S. Wainger, 'On Hilbert transforms along curves, II', Amer. J. Math. 98 (1976), 395-403.

[10] F. Ricci and G. Weiss, 'A characterization of $H^{1}\left(\Sigma_{n-1}\right)$ ', in: Harmonic Analysis and Euclidean Spaces, Proceedings of Symposia in Pure Mathematics, 35 (American Mathematical Society, Providence, RI, 1979), pp. 289-294.

[11] E. M. Stein and G. Weiss, Introduction to Fourier Analysis on Euclidean Spaces (Princeton University Press, Princeton, NJ, 1971).

YANPING CHEN, School of Mathematical Sciences, Beijing Normal University, Beijing 100875, China

Applied Science School, University of Science and Technology,

Beijing 100083, China

e-mail: yanpingch@126.com

YONG DING, School of Mathematical Sciences, Beijing Normal University, Laboratory of Mathematics and Complex Systems, Ministry of Education,

Beijing 100875, China

e-mail: dingy@bnu.edu.cn 
DASHAN FAN, Department of Mathematics, University of Wisconsin-Milwaukee, Milwaukee, WI 53201, USA

Huazhong Normal University, Wuhan 430074, China

e-mail: fan@uwm.edu 